\title{
Fabrication of Liquid Molds using Drop-on-demand Printing Technology for Bio-Pdms Mirofluidic Devices
}

\author{
Lijun Yang*, Baochun Lu, Li Zhu, and Xiaoyang Zhu
}

Microsystem Lab, School of Mechanical Engineering, Nanjing University of Science and Technology, Nanjing, Jiangsu, China

\begin{abstract}
A simple and easy method is demonstrated for the fabrication of liquid molds which was used for the fabricaion of bio-polydimethylsiloxan (Bio-PDMS) mirofluidic devices based on a novel drop-on-demand (DOD) printing technology. The liquid molds were DOD printed well on the hydrophilic glass substrate which was treated with a $\mathrm{TiO}_{2}$ nanoparticles $\left(\mathrm{TiO}_{2} \mathrm{NPs}\right.$ ) solution at the overlap of $30 \%$. Then the PDMS concave molds were fabricated well by being replicated from the liquid molds and were bonded with another PDMS substrate to form a Bio-PMDS mirofluidic device. The micro-channel which the width and the height were about $100 \mu \mathrm{m}$ and $8 \mu \mathrm{m}$ was fabricated and the surface roughness of the micro-channel with the $100 \times 320 \mu \mathrm{m}^{2}$ area was about $179 \mathrm{~nm}$ measured by a white light interferometer. The experimental results showed that the width of micro-channel in the Bio-PDMS microfluidic device was small and the surface of the micro-channel was smooth.
\end{abstract}

Keywords: Liquid molds; Drop-on-demand printing; Bio-PDMS microfluidic device; Hydrophilic; Overlap

\section{Introduction}

In the last few decades, biological microfluidic devices have been attracting considerable interests in the area of biosensors and bioelectronics due to the characteristics of less consumption of samples, less analysis time and portability [1]. Recently, there have been many studies on biosensors and bioelectronics using biological mircofluidic devices [2,3]. Polydimethylsiloxane (PDMS), a silicon-based polymer, is often the preferred material for the prototyping of microfluidic devices as it is easily to be bonded, transparent ,durable, non-fluorescent, biocompatible and nontoxic $[4,5]$. The most widely used method for PDMS microfluidic device fabrication is soft lithography. However, the fabrication of master molds is the key step of PDMS microfluidic device preparation with a soft lithography method.

To date, there have been many methods developed to fabricate master molds in bio-PDMS mirofluidic devices such as, UV exposure, ice-water patterning [6], the etching of copper, liquid molding on paper [7] and wax printing on paper [8]. However, most of these methods have some limitations, making the fabrication expensive, complex and not easy. The UV exposure on photosensitive polymers needs photomasks and organic solvent [9], and the equipment was complicated and the fabrication must be carried in an ultra-clean environment. The etching of copper required masks and additional etching steps [10], and the solution needed in the fabrication was toxic. Liu X fabricated PDMS micro-devices with a "liquid-molding" method [11], but however, the process is complex and difficulty. Because the method needs to photo-lithographically fabricate micro-patterns on a silanized glass substrate to form hydrophilic/hydrophobic surfaces and to fabricate 3D patterns of a liquid via dip-coating the substrate in a polar solution. We prepared a PDMS microfluidic device based on drop-on-demand generation of wax molds [12], in which the wax droplets were dispersed on the substrate to form the wished patterns at the desired times and positions without contact with the substrate. However, the surface of the micro-channels in the micofuidic devices was rough and the width of the channel was large.

In this paper, a new droplet-on-demand printing method [13-15] was described to fabricate liquid molds which were used in Bio-PDMS microfluidic devices on a hydrophilic glass substrate using a glycerol solution. The liquid droplets were ejected from a glass micro-nozzle onto the glass substrate based on microfludic pulse interior force to form different liquid molds. Finally, the depth, the width and the surface roughness of the micro-channel were characterized.

\section{Materials and Method}

Glycerol AR was purchased from Sinopharm Chemical Reagent Co., Ltd. Sylgard 184 was supplied by the Dow Corning Corporation. The $\mathrm{TiO}_{2}$ nanoparticals(NPs) with the diameter of less than $4 \mathrm{~nm}$ was purchased from Shenzhen jing cai chemi cal co.,ltd. The borosilicate glass capillary $(1.0 \mathrm{~mm} \times 0.6 \mathrm{~mm} \times 100 \mathrm{~mm})$ was purchased from Beijing Zhengtianyi Scientific And Trading Co., Ltd. The micro-nozzles used in this paper was made by a Microelectrode puller (MODEL P-2000) and a platinum resistance wire (MF-900.NARISHIGE) with the borosilicate glass capillary to get the outlet inner diameters. Figure 1 shows the fabrication process of the micro-nozzles with the platinum resistance wire in four steps. Firstly, the glass capillary tip was placed above the glass micro-ball. Then the glass micro-ball was heated by controlling the voltage and the tip was cut off at the desired position. Afterward, the tip was placed in front of the glass micro-ball. At last, the tip was forged to form a micro-nozzle by heating the micro-ball through controlling the voltage.

In the DOD printing system, as is shown in Figure 2, a glass micronozzle filled with the $50 \%$ glycerol solution is fixed with the PZT actuator through a connecting device. The liquid in the micro-nozzles was jetted on the glass substrate to form liquid droplets with different diameters by the pulse inertia force supplied by a PZT actuator. The glass substrates used in this paper were hydrophilic treated by $\mathrm{TiO}_{2}$

*Corresponding author: Lijun Yang, Microsystem Lab, School of Mechanica Engineering, Nanjing University of Science and Technology, Nanjing, Jiangsu, China, Tel: +86-025-84315031; E-mail: fulisayang@163.com

Received October 05, 2015; Accepted October 12, 2015; Published October 21 2015

Citation: Yang L, Lu B, Zhu L, Zhu X (2015) Fabrication of Liquid Molds using Drop-on-demand Printing Technology for Bio-Pdms Mirofluidic Devices. J Biosens Bioelectron 6: 185. doi:10.4172/2155-6210.1000185

Copyright: (c) 2015 Yang L, et al. This is an open-access article distributed under the terms of the Creative Commons Attribution License, which permits unrestricted use, distribution, and reproduction in any medium, provided the original author and source are credited. 


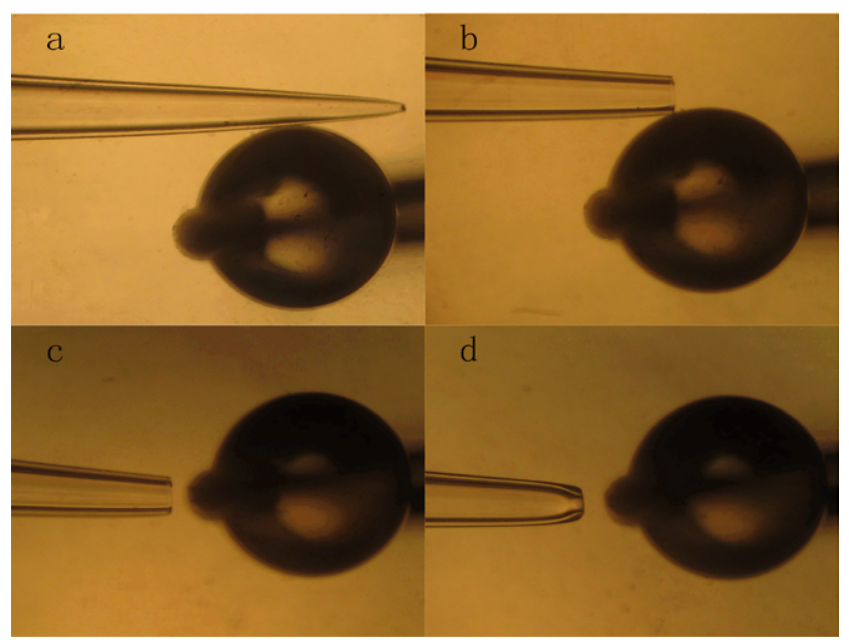

Figure 1: Typical response of sensors to sample air and classification features: vA) minimum value of the ascending curve; vB) maximum value of the ascending curve; $\mathrm{vC}$ ) minimum value of the descending curve.

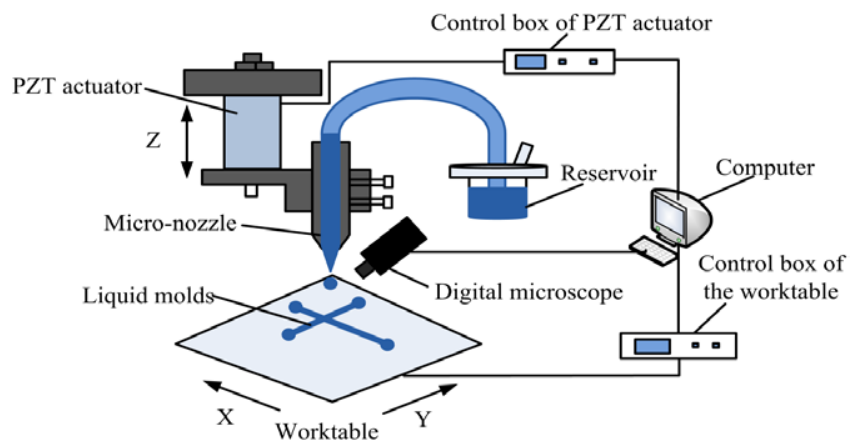

Figure 2: Schematic of liquid jetting system based on the digitallization of microfluidic technology.

NPs solution. The higher the $\mathrm{TiO}_{2}$ NPs concentration is, the more hydrophilic the substrate is. The liquid lines can be formed on the hydrophilic glass substrate by changing the overlap of the liquid droplets using a computer-controlled motorized X-Y worktable.

Figure 3 shows the fabrication process of the bio-PDMS microfluidic device. Firstly, the glass substrate was cleaned and hydrophilic treated with $\mathrm{TiO}_{2}$ NPs solution, as is shown in Figure $3 \mathrm{a}$ and $3 \mathrm{~b}$, then the $50 \%$ glycerin solution was printed on the substrate to form liquid molds, as is shown in Figure 3c. Afterward, the PDMS liquid was poured on the liquid molds and been put in a drying oven for 12 hours at $60^{\circ} \mathrm{C}$, as is shown in Figure 3d. The PDMS concave mold was peeled off from the substrate and drilled after being cured, as is shown in Figure 3e. At last, the PDMS device was bonded with another PDMS by pressing and maintaining at $75^{\circ} \mathrm{C}$ for half an hour, as is shown in Figure $3 \mathrm{f}$.

\section{Results and Discussion}

Figure 4 shows the micrographs of liquid droplet arrays with different diameters on the hydrophilic substrates. The result showed that the glycerol liquid could be jetted steadily on the hydrophilic glass substrate and the liquid droplet arrays had good consistency. The driving voltage was $40 \mathrm{~V}$ and the frequency was $2 \mathrm{~Hz}$. The distance between the neighboring droplets was $200 \mu \mathrm{m}$. The outlet inner diameters of the micro-nozzles used in Figure $4 \mathrm{a}-\mathrm{c}$ were respectively 80 $\mu \mathrm{m}, 60 \mu \mathrm{m}$ and $40 \mu \mathrm{m}$. The diameter of the liquid droplets is controlled by the outlet inner diameter of glass micro-nozzles, the driving voltage and the driving frequency of the microfluidic system. The droplet diameters became larger when the driving voltages, driving frequencies and the micro-nozzle outlet inner diameters increased.

The surface treatments of the glass substrates and the overlap of the neighboring droplets play a great role in the formation of the liquid lines. When the substrate is hydrophobic or less hydrophilic, the neighboring droplets would like to get together to bump into a ball, as is shown in Figure 4a. The overlap of the droplets can be neither too large, nor too small. When the overlap is too small, the droplets were separated and unable to link together. However, when the overlap is too large, such as $60 \%$, line bulges would be produced, as is shown in Figure $4 \mathrm{~b}$. When the overlap of the substrate is $30 \%$, the liquid lines seemed to be well formed on a hydrophilic substrate, as is shown in Figure 4c.

After fabricating liquid molds on the hydrophilic substrates, the PDMS concave mold and the Bio-PDMS microfludic device were fabricated in several steps as above (Figure 5). Then, the characters of the concave mold were measured. Figure 6 shows the SEM photo and the three dimensional surface profile of a PDMS micro-channel replicated form a liquid mold. The width and the height of the channel were about $100 \mu \mathrm{m}$ and $8 \mu \mathrm{m}$. The surface smoothness of PDMS microchannels replicated form liquid molds was measured by a white light interferometer. The surface roughness of the micro-channels was about $179 \mathrm{~nm}$, as is shown in Figure 7. The surface of micro-channels fabricated in the Bio-PDMS microfluidic device was smooth.

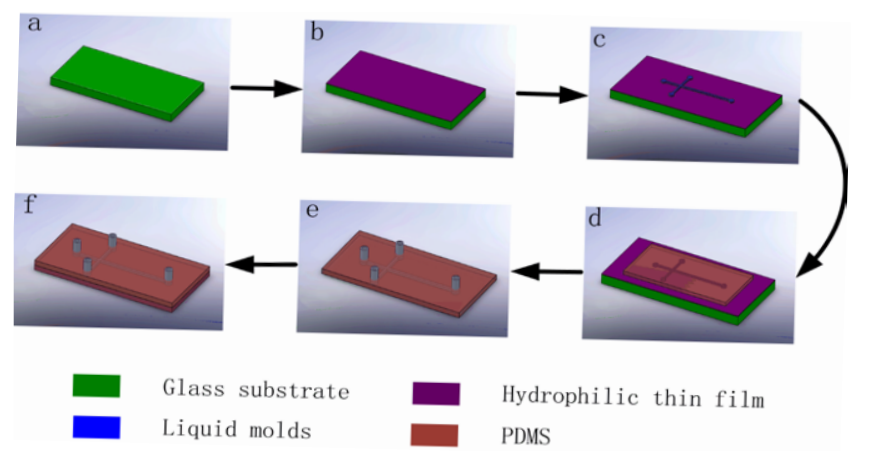

Figure 3: Fabrication process of the Bio-PDMS microfluidic device:(a) untreated, but cleaned glass substrate; (b)hydrophilic treated glass substrate; (c)DOD printing liquid mold on the treated glass substrate; (d)pouring the PDMS on the liquid mold and cured; (e)peeling off the cured PDMS concave mold from the substrate and drilling (f) the PDMS concave mold was bonded with a PDMS substrate.

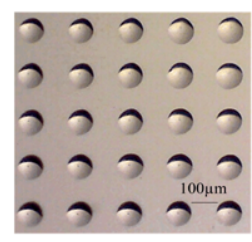

(a)

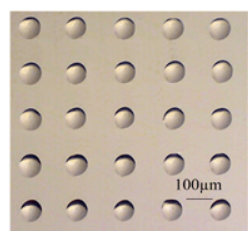

(b)

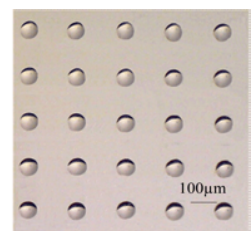

(c)
Figure 4: Micrographs of the liquid droplets with different diameters. 
Citation: Yang L, Lu B, Zhu L, Zhu X (2015) Fabrication of Liquid Molds using Drop-on-demand Printing Technology for Bio-Pdms Mirofluidic Devices. J Biosens Bioelectron 6: 185. doi:10.4172/2155-6210.1000185

Page 3 of 3

$\mathrm{a}$

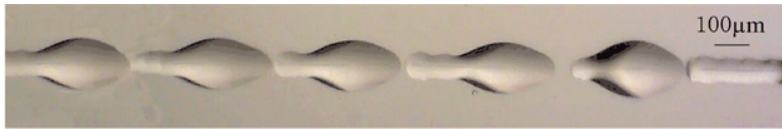

b

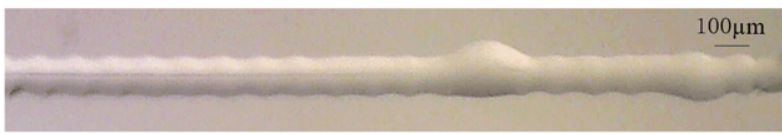

C

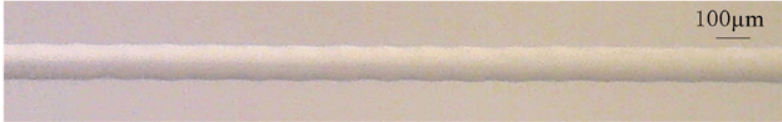

Figure 5: (a) Microscopic image of liquid line on the less hydrophilic substrate at a $30 \%$ overlap; (b) Microscopic image of liquid line on the hydrophilic substrate at a $60 \%$ overlap; (c) Microscopic image of liquid line on the hydrophilic substrate at a $30 \%$ overlap.

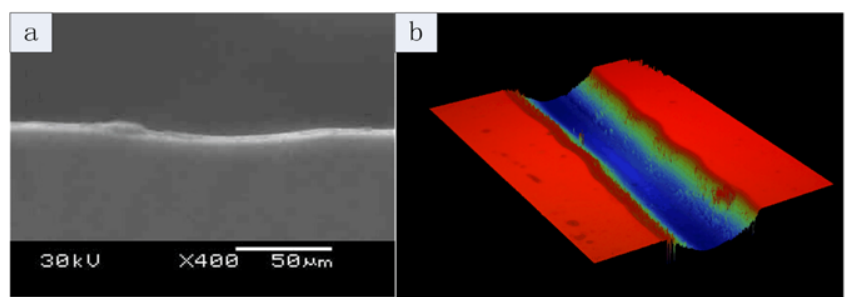

Figure 6: (a) SEM photo of a PDMS micro-channel replicated form a liquid mold; (b) the three dimensional surface profile of a PDMS micro-channel replicated form a liquid mold.

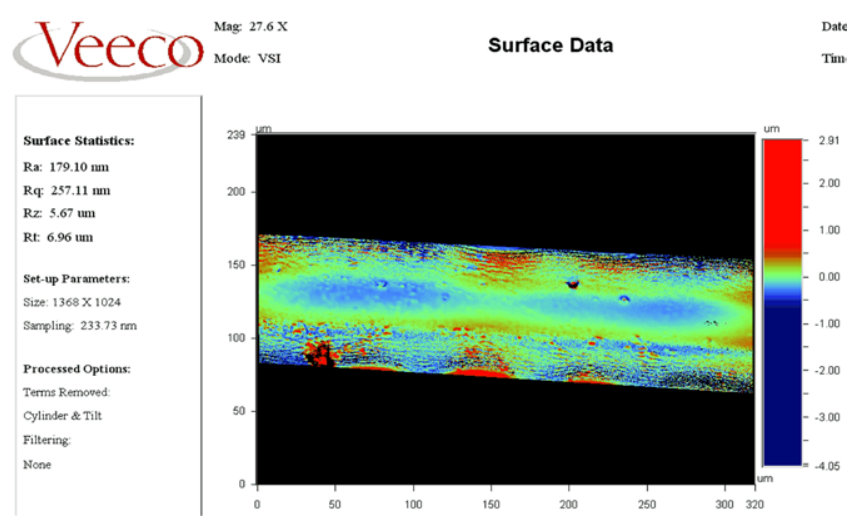

Figure 7: The surface smoothness of PDMS micro-channel replicated form a liquid mold by white light interferometer.

\section{Conclusion}

The experiment results showed that based on the drop-ondemand technology, the glycerol liquid could be jetted steadily on the hydrophilic glass substrate, and the diameter of the liquid droplets was controlled by the outlet inner diameter of glass micro-nozzles, the driving voltage and the driving frequency of the microfluidic system. With the overlap of $30 \%$ on the hydrophilic substrate, the liquid line seemed to be well formed. After that, the PDMS concave molds for Bio-PDMS device were well fabricated. The width and the height of the micro-channel were about $100 \mu \mathrm{m}$ and $8 \mu \mathrm{m}$. The surface roughness of the micro-channel was about $179 \mathrm{~nm}$. The liquid molds printing system is simple structured, the glass micro-nozzles are easily fabricated and low cost. This method suggests a novel path which is easy, inexpensive and portable in the area of biological microfluidic devices fabrication.

\section{Acknowledgements}

This work is supported by the National Natural Science Foundation of China (NO.51175268, NO.11102090).

\section{References}

1. Arora A, Simone G, Salieb-Beugelaar GB, Kim JT, Manz A (2010) Latest developments in micro total analysis systems. Anal Chem 82: 4830-4847.

2. Radke SM, Alocilja EC (2004) Design and fabrication of a microimpedance biosensor for bacterial detection J. Sensors Journal, IEEE 4: 434-440.

3. Cen EG, Qian L, Kaler KVIS (2003) An integrated microchip for dielectrophoresis based characterization and manipulation of cells. Engineering in Medicine and Biology Society, Proceedings of the 25th Annual International Conference of the IEEE. IEEE 4: 3344-3347.

4. Fiorini GS, Chiu DT (2005) Disposable microfluidic devices: fabrication function, and application. Biotechniques 38: 429-446.

5. Becker H, Gärtner C (2008) Polymer microfabrication technologies for microfluidic systems. Anal Bioanal Chem 390: 89-111.

6. Waldbaur A, Rapp H, Länge K, Bastian E (2011) Let there be chip-towards rapid prototyping of microfluidic devices: one-step manufacturing processes. Analytical Methods 12: 2681-2716.

7. Browne AW, Rust MJ, Jung W, Lee SH, Ahn CH (2009) A rapid prototyping method for polymer microfluidics with fixed aspect ratio and $3 \mathrm{D}$ tapered channels. Lab Chip 9: 2941-2946.

8. Koesdjojo MT, Koch CR, Remcho VT (2009) Technique for microfabrication of polymeric-based microchips from an SU-8 master with temperature-assisted vaporized organic solvent bonding. Anal Chem 81: 1652-1659.

9. Lu Y, Lin B, Qin J (2011) Patterned paper as a low-cost, flexible substrate for rapid prototyping of PDMS microdevices via "liquid molding". Anal Chem 83 1830-1835.

10. Kaigala GV, Ho S, Penterman R, Backhouse CJ (2007) Rapid prototyping of microfluidic devices with a wax printer. Lab Chip 7: 384-387.

11. Liu X, Wang Q, Qin J, Lin B (2009) A facile "liquid-molding" method to fabricate PDMS microdevices with 3-dimensional channel topography. Lab Chip 9: 1200-1205.

12. Li Z, Hou L, Zhang W, Zhu L (2014) Preparation of PDMS microfluidic device based on drop-on-demand generation of wax molds. Analytical Methods 6: 4716-4722.

13. Zhang W, Hou L (2005) Science and Technology Review 23: 4-9.

14. Zhang W, Hou L (2006) Science and Technology Review 24: 41-47.

15. Zhu X, Zhu L, Chen H (2015) Fabrication of high numerical aperture micro-lens array based on drop-on-demand generating of water-based molds. Optics and Laser Technology 68: 23-27. 